\title{
Influences of affiliated components and train length on the train wind
}

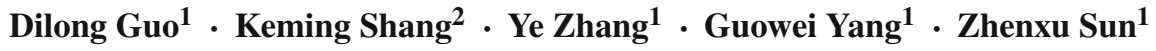

Received: 30 July 2015 / Revised: 28 October 2015 / Accepted: 2 November 2015 / Published online: 17 March 2016

(C) The Chinese Society of Theoretical and Applied Mechanics; Institute of Mechanics, Chinese Academy of Sciences and Springer-Verlag Berlin Heidelberg 2016

\begin{abstract}
The induced airflow from passing trains, which is recognized as train wind, usually has adverse impacts on people in the surroundings, i.e., the aerodynamic forces generated by a high-speed train's wind may act on the human body and endanger the safety of pedestrians or roadside workers. In this paper, an improved delayed detached eddy simulation (IDDES) method is used to study train wind. The effects of the affiliated components and train length on train wind are analyzed. The results indicate that the affiliated components and train length have no effect on train wind in the area in front of the leading nose. In the downstream and wake regions, the longitudinal train wind becomes stronger as the length of the train increases, while the transverse train wind is not affected. The presence of affiliated components strengthens the train wind in the near field of the train because of strong flow solid interactions but has limited effects on train wind in the far field.
\end{abstract}

Keywords Train wind - Simplified model - Real train model $\cdot$ Longitudinal train wind $\cdot$ Transverse train wind . Affiliated components

Dilong Guo

jack9517@126.com

1 Key Laboratory for Mechanics in Fluid Solid Coupling Systems, Institute of Mechanics, Chinese Academy of Sciences, No. 15 Beisihuanxi Road, Beijing 100190, China

2 CSR Qingdao Sifang Co. Ltd., Qingdao 266111, China

\section{Introduction}

Railway transportation is the most efficient method of high-speed transportation in terms of energy savings and environmental protection, compared with the automobile or aircraft. Based on statistics released by Japan's Ministry of International Trade and Industry, the energy consumption required for a $1 \mathrm{~km}$ shift per person are approximately 1:4:6 for trains, planes, and cars respectively, and that of carbon dioxide emissions is 1:6:10. Therefore, in densely populated countries, such as China, Japan, and European countries, many high-speed railways were built. The operations of the high-speed trains will inevitably have certain impacts on the environment, including, for example, noise or train wind. Train wind is the flow induced around a running train, and the disturbances caused by the changes in air pressure and velocity will affect the trackside workers and passengers on the platform and might also roll up the goods on the trackside or a platform [1-3]. The magnitude of train wind is proportional to the running speed of the train. It can be imagined that the accident risks for trackside workers and passengers on a platform rise as the running speed of the train increases.

When a train is running, there are three regions where airflow can be induced along the train's path of travel $[4,5]$. The first region is in front of the leading nose: when the train moves forward, the air ahead of the leading nose is pushed forward and outward, which forms a so-called bow compression wave in front of the train nose and causes train wind. The second region is on the sides of the train. In this region, the air is attached to the surface of the train, and a boundary layer is formed between the train surface and the surrounding air owing to air viscosity, leading to a strong velocity gradient. The third region is the wake region at the rear of the train, 
which consists of a complex vortex field with turbulent flows $[6,7]$. Therefore, people and objects close to a train passing at high speed could experience high wind-generated forces [8]. Airflows in the first region can be considered inviscid flows, which can be solved using potential flow theory or a panel method [9]. For train wind in the second region, the flow field has normally been solved by means of boundary-layer theory. For the wake region, the flow field can be solved by means of a discrete-vortex method [10,11]. The train wind ahead of the leading nose is affected by the shape of the train head [12]. The blunter the shape of the train head, the greater the force generated by the train wind and the shorter the actuation duration. The thickness of the boundary layer around the train is mainly a function of the length of the train, bogies, windshield, and other areas of the train. The projections and grooves on the train surface cause perturbations in the local flow field, which lead to variations in the boundary-layer thickness and the flow characteristics inside the boundary layer; consequently, the distribution of the train wind will vary. Obviously, it is more reasonable to use boundary-layer theory to analyze the flows on the lateral sides of a train. The strength of the train wind inside the boundary layer decreases as the cross distance to the center of the train (COT) increases and also decreases as the height from the ground increases. The wake of a high-speed train is characterized by shear layers, von Karman-type vortex sheddings, separation and recirculation regions, and a pair of twin counterrotating longitudinal vortices. When a steep shape is adopted for the rear surface of the trailing nose, airflows separate at the top and side surfaces of the train, forming a separation bubble. When a flat shape is adopted for the rear surface of the trailing nose, airflows reattach on the rear surface and are accelerated downward, forming a low-pressure region. The airflows separate over the side edges and are curled into two counterrotating vortices, resulting in much higher drag than in a separation bubble $[7,13,14]$.

Two methods are used to evaluate the magnitude of train wind. In Japan and England, the average train wind near people is adopted as the criterion, and the value is $9 \mathrm{~m} / \mathrm{s}$ in Japan and $11.1 \mathrm{~m} / \mathrm{s}$ in England for platform safety. In France and Germany, the aerodynamic forces acting on the human body are used as the criterion, and the allowable maximum value is $100 \mathrm{~N}$ [15]. In the previous scaled model test and numerical simulations, a simplified model was used to study train wind with a 3-car grouping. The affiliated components, such as the bogie, bogie cabin, and windshield, were not included in the train model $[12,16]$. The human body model was also simplified to a cylinder. Although these affiliated components do not affect the bow wave ahead of the leading nose, they will affect the development and the formation of the boundary layer and the wake. Therefore, it is not accurate to study train wind using a simplified model. In the present study, a real train model is used to study train wind. The effects of affiliated components and the length of the train model on the train wind are analyzed.

\section{Computational model and domain}

The train models adopted in this paper are full-scale CRH380A trains, which are widely used in the Chinese railway transit system. The models include simplified models with various groupings and the real models. In real train models, bogies, bogie cabins, and windshields are included. The grouping type includes 3-car, 8-car and 16-car models, respectively. The length of each car is approximately $26 \mathrm{~m}$, while the width of the car body is $3.38 \mathrm{~m}$, and the distance between the ground and the wheel flange is $0.176 \mathrm{~m}$. The height of the train is denoted by a characteristic length $H$ of $3.5 \mathrm{~m}$. The computational domain extends $30 \mathrm{H}$ ahead of the train nose and $60 \mathrm{H}$ from the train tail to the exit of the computational domain. The top boundary of the computational domain is at a distance of $30 \mathrm{H}$ from the bottom of the rail and the side boundaries are at a distance of $30 \mathrm{H}$ from the center axis of the train. An outline of the computational domain and the model are shown in Fig. 1. The computational domains for all train models with different car groupings are identical.

\section{Numerical detail}

The commercial computational fluid dynamics (CFD) code STAR-CCM+ was utitilized to simulate the air flows generated by passing trains [17]. The STAR-CCM+ code integrates the preprocessor, the CFD solver, and the postprocessor into a package and can generate high-quality meshes for complex geometries. The STAR-CCM+ code supports various meshing strategies that are suitable for different applications. In this study, a trimmed cell mesher was selected to generate the volume mesh and to resolve the detailed flows adjacent to the train surface. Three mesh density zones were specified to refine the volume meshes. In each mesh density zone, the mesh size was $0.08,0.16$, and $0.64 \mathrm{~m}$. To resolve the surface boundary layer, a prism layer of ten cells was created in a belt of thickness $0.02 \mathrm{~m}$ around the train, with a stretching ratio of 1.2. The height of the first layer mesh was about $8 \times 10^{-4} \mathrm{~m}$, which ensures that $Y+$ ranges from 30 to 100 . The boundary-layer cells were generated using the prism layer mesher. Figure 2 depicts the cut section of the train model with an 8-car grouping on the symmetry plane. The surface grids on the train head, bogie, and windshield are shown in Fig. 2b-d, respectively. The total number of volume cells was 56 million for the 8-car real train model, and the volume cell counts of the other two types of real train models are 22 and 96 million, respectively. The vol- 

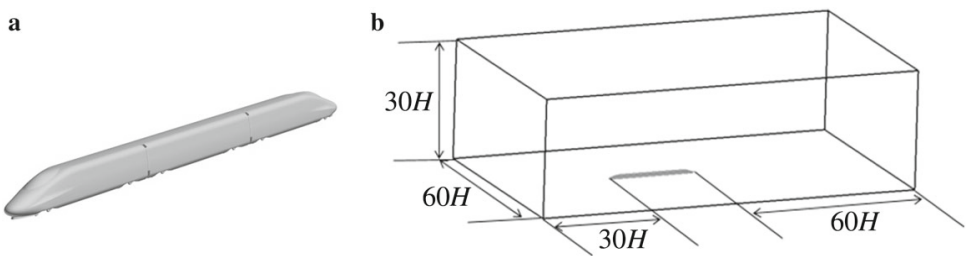

Fig. 1 a Train model. b Outline of computational domain

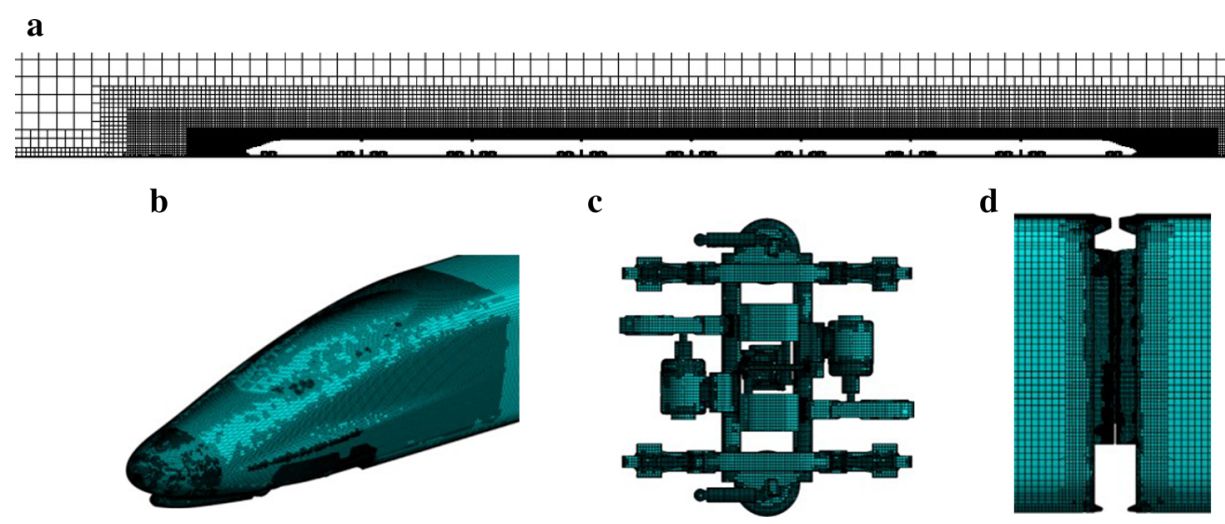

Fig. 2 Computational mesh. a Section mesh in symmtry plane. b Train head surface mesh. c Bogie surface mesh. d Windshield surface mesh

ume cell counts for the three types of simplified model are 11, 20, and 39 million, respectively. The simulations consumed a lot of computational resources. All the calculations were carried out in a high-performance parallel computing large computer cluster at the Supercomputing Center of the Chinese Academy of Sciences.

The airflows generated by running trains are very complex and are characterized by unsteady turbulence flows. In this study, an improved delayed detached eddy simulation (IDDES) formulation is used to accurately simulate the details of flow fields. This method combines DDES with an improved Reynolds-averaged Navier-Stokes equations-large eddy simulation (RANS-LES) hybrid model for wall modeling in LES when the grid resolution is sufficient. Considering the running speed of a train, a coupled implicit unsteady solver is used. The inviscid flux term is discretized using a Weiss-Smith preconditioned Roe's flux-difference splitting scheme. The transient term is discretized in an Euler implicit second-order temporal scheme. The dual time-stepping scheme is used for solving unsteady flows with a physical time-step size of $0.001 \mathrm{~s}$ and five subiterations per time step.

Free-stream boundary conditions are applied to the inlet, exit, top, and side boundaries of the computational domain. The no-slip wall boundary is set for the ground with a moving velocity identical to the running speed of the train, which is
$97.22 \mathrm{~m} / \mathrm{s}(350 \mathrm{~km} / \mathrm{h})$. The turbulent initial conditions are based on a synthetic eddy method, the turbulence intensity is 0.01 , and the turbulent length scale is $0.01 \mathrm{~m}$.

\section{Validation}

Experimental measurements were performed to validate the mesh strategy and the numerical method. The test model used in the measurement is a $1 / 8$ th scaled model of the modified CRH380A train with a 3-car grouping, including the train head, intercar, and train tail, and the length of the model is $9.75 \mathrm{~m}$. The experiment was conducted at the Shanghai Automotive Wind Tunnel Center in Tongji University. The free stream velocity is $60 \mathrm{~m} / \mathrm{s}$. The mesh strategy and numerical method utilized in the validation are the same as described in Sect. 3, and the total number of mesh cells is approximately 24 million. The experimental setup in the wind-tunnel and the mesh of the model are shown in Fig. 3a, b. The positions of the pressure monitors on the head train surface are shown in Fig. 4 and are located on the symmetry plane of the model. The same monitor distributions can be found for the tail train in reverse sequence. The numerical results are the time-averaged values over a $2 \mathrm{~s}$ period after the flows are fully developed and are normalized by the free stream reference values. It can be seen that the numerical predictions 

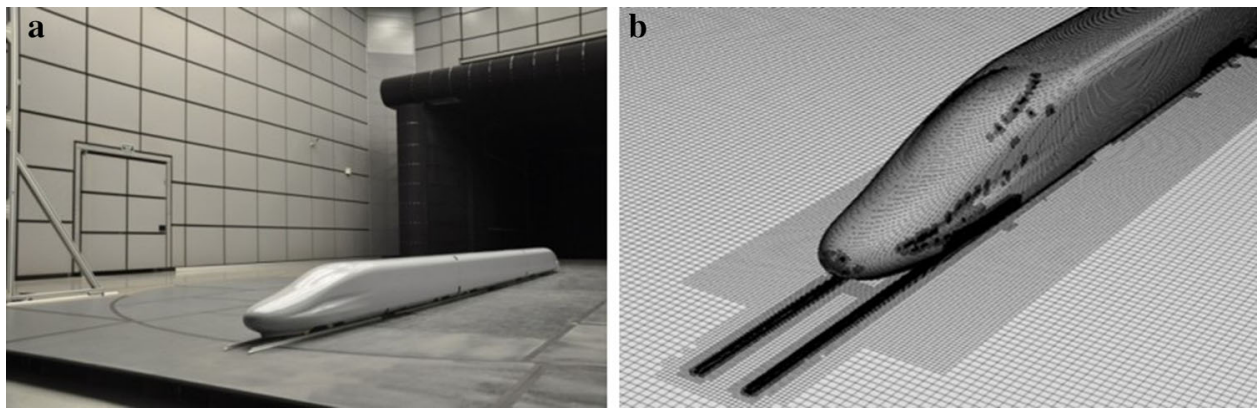

Fig. 3 a Photograph of wind tunnel model. b Surface mesh of train model and ground

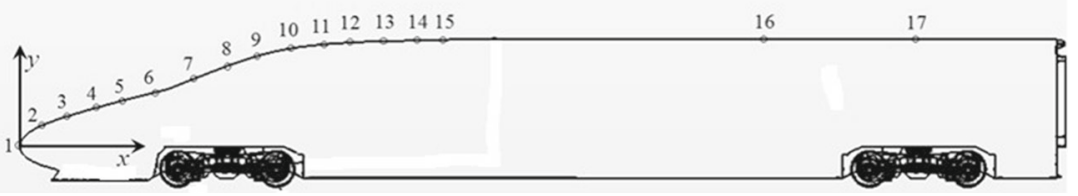

Fig. 4 Schematic diagram of measuring point
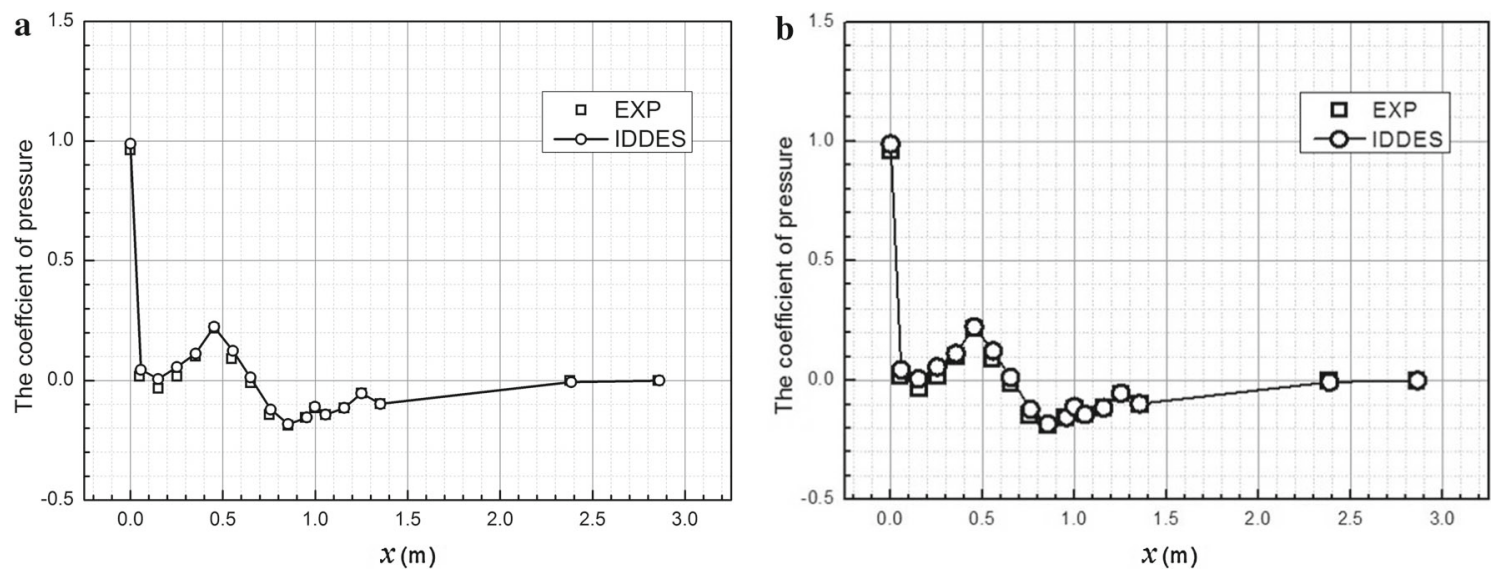

Fig. 5 a Comparison between experimental and numerical results in train head. b Comparison between experimental and numerical results in train tail

are in good agreement with the experimental values, which indicates that the mesh strategy and numerical method used in this paper are reasonable (Fig. 5).

\section{Results and discussion}

The purpose of this study is to analyze the effects of the length of a train and the affiliated components on train wind. The results are presented in two sections. First, the characteristics of train wind are analyzed when simplified train models with 3-, 8-, and 16-car groupings are used. In the second section, the characteristics of train wind are analyzed when real train models are used. The effect of the affiliated components on train wind is analyzed by a comparison between the simplified and real train models. Owing to the high unsteadiness of the flows around the trains, the velocity of the train wind will be presented with the time-averaged values over a $2 \mathrm{~s}$ period.

\subsection{Characteristics of train wind for simplified train model}

In this study, the train is stationary when the flow field is calculated, the free stream and the ground have the velocity of a running train, and therefore, when analyzing the train wind, stationary ground should be taken as the reference frame. The reference frame is established as follows: the direction of the train movement is the $x$-axis positive direction, the left side of the train movement is the $y$-axis positive direction, and the $z$-axis is determined in accordance with a right-handed coordinate system. The coordinates of the tip of the train's leading nose are $x=-39 \mathrm{~m}, y=0 \mathrm{~m}$, and $z=1 \mathrm{~m}$. The length of the streamline shape of the train head is $12 \mathrm{~m}$. 

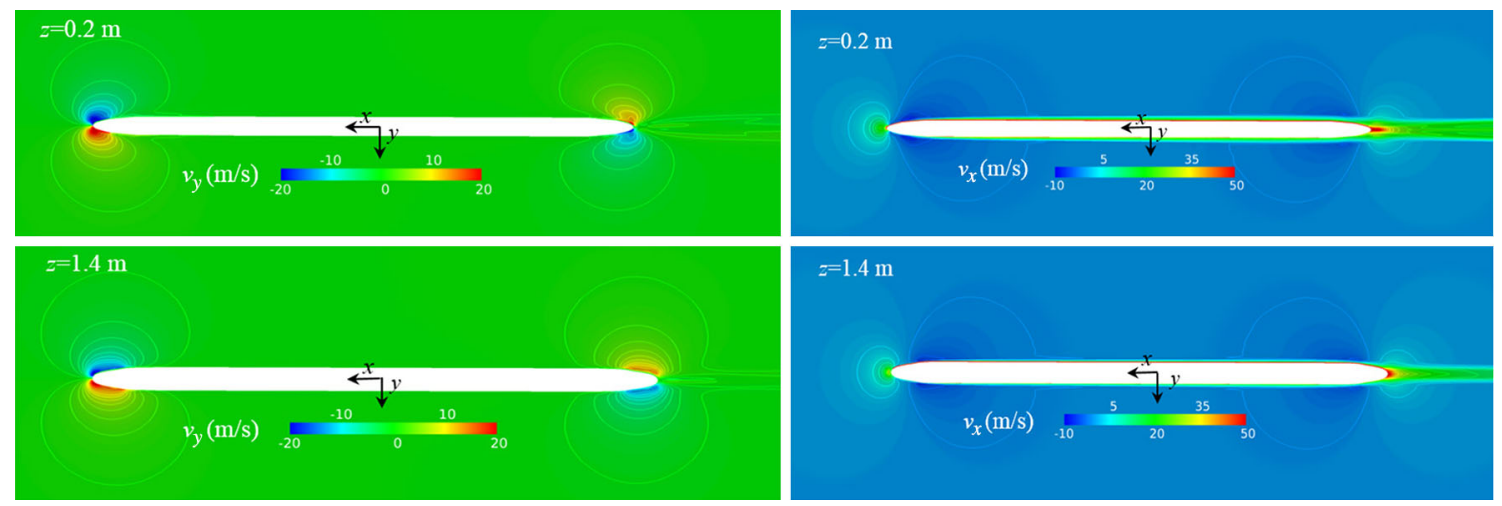

Fig. 6 Contours of velocity of transverse train wind (TTW) and longitudinal train wind (LTW) in plane $z=0.2 \mathrm{~m}$ and $z=1.4 \mathrm{~m}$
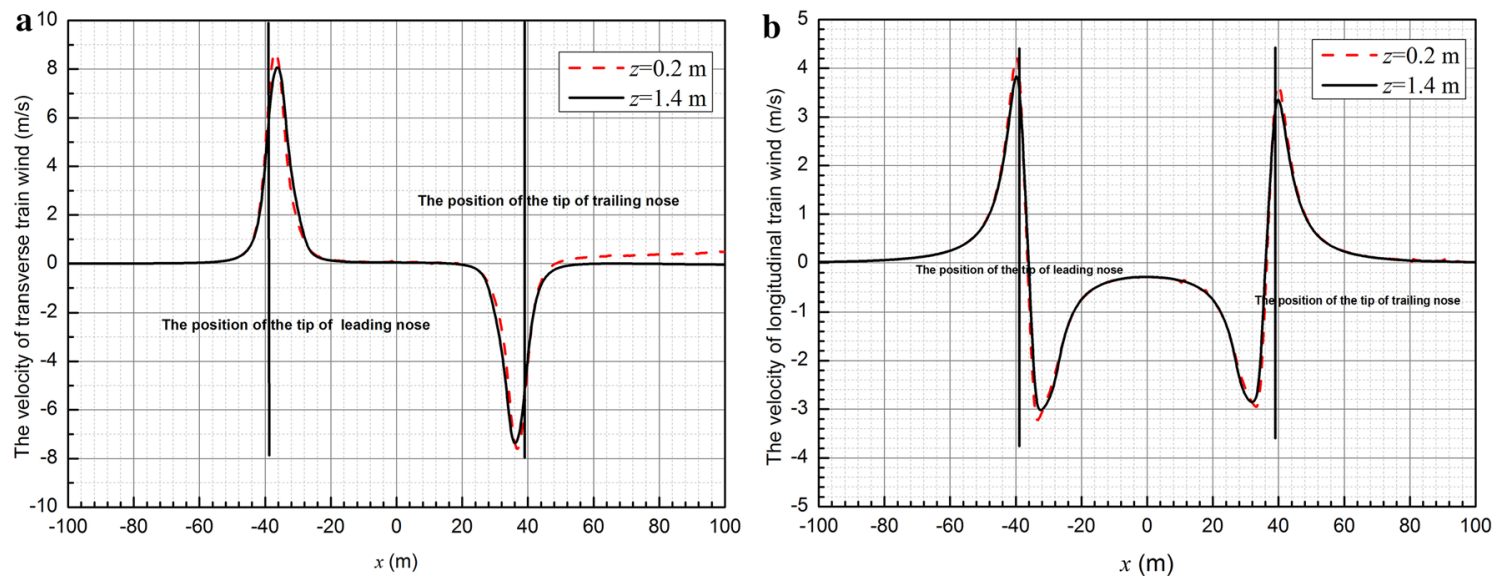

Fig. 7 a TTW of 3-car group simplified train along train length. b LTW of 3-car group simplified train along train length

When the train moves ahead, the airflow ahead of the leading nose is pushed forward and laterally, airflows that are just upstream and transverse change dramatically because of the cross-sectional shape of the varying train head. In the middle of the train, the cross-sectional shape of the train remains constant, and the airflow changes slightly. In the train's trailing nose region and wake region, since the airflow is affected by the separated and detached flow near the trailing nose and the counterrotating vortices behind the trailing nose, the airflow changes dramatically, too.

In Fig. 6, the longitudinal (train movement direction) and transverse velocity of airflow around the train in the plane $z=0.2 \mathrm{~m}$ and $z=1.4 \mathrm{~m}$ are shown. It can be seen that the train wind changes dramatically in the nose and wake regions. In Fig. 7, the longitudinal and transverse velocities of train wind along the train length are illustrated at $3.0 \mathrm{~m}$ from the center of the train at different distances from the ground. The measuring position is based on BS EN 14067. It can be seen that the maximum longitudinal train wind (LTW) is below the maximum transverse train wind (TTW).
The train wind in a lower position is greater than that in a higher position. In the case of TTW, when the train is passing, the train wind velocity ahead of the tip of the leading nose increases dramatically along the train, then decreases behind the leading nose. The maximum velocity of the TTW appears at the position $x=-38 \mathrm{~m}$, which is $1 \mathrm{~m}$ behind the leading nose. The TTW is approximately $0 \mathrm{~m} / \mathrm{s}$ in the middle of the train. In the train's trailing nose and wake regions, the airflow is sucked toward the trailing nose, and the maximum TTW appears at the position $x=36 \mathrm{~m}$, which is $3 \mathrm{~m}$ ahead of the trailing nose. In the case of LTW, the train wind velocity increases and then decreases dramatically in the nose region. Two peaks can be observed sequentially for the leading nose region, a positive peak and a negative peak. As for the former, the velocity of the train wind increases in the running direction, reaches its maximum at the tip of the leading nose, and then decreases dramatically. As for the latter, the airflow runs in the opposite direction of the train and reaches its maximum around the streamline, while the converse is true in the trailing nose and wake regions. The maximum LTW 


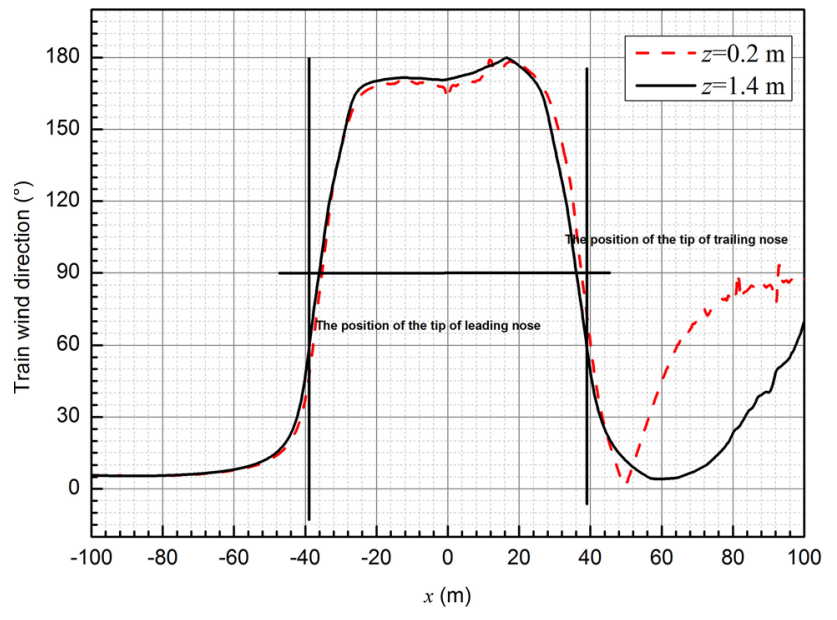

Fig. 8 Train wind direction at $3.0 \mathrm{~m}$ from COT at different heights from ground along 3-car group simplified train length

appears at the position $x=40$ ( $1 \mathrm{~m}$ behind the tip of the trailing nose). The train wind in the transverse direction is greater than that in the longitudinal direction. Especially in the leading nose and trailing nose regions, a relatively large train wind appears. The variation in the cross section of the streamlined shape could draw in a stronger train wind. For the position $3 \mathrm{~m}$ away from the train center, the boundary-layer effect seems to influence the train wind to a lesser extent. For the simplified train model, the train wind caused by the leading nose is slightly greater than that caused by the trailing nose.

Figure 8 shows the variation in the train wind direction at $3.0 \mathrm{~m}$ from the COT at 0.2 and $1.4 \mathrm{~m}$ high from the ground along the train length. It can be seen that the directions are outward along the leading streamline, backward along the train body, and inward and forward along the trailing streamline.

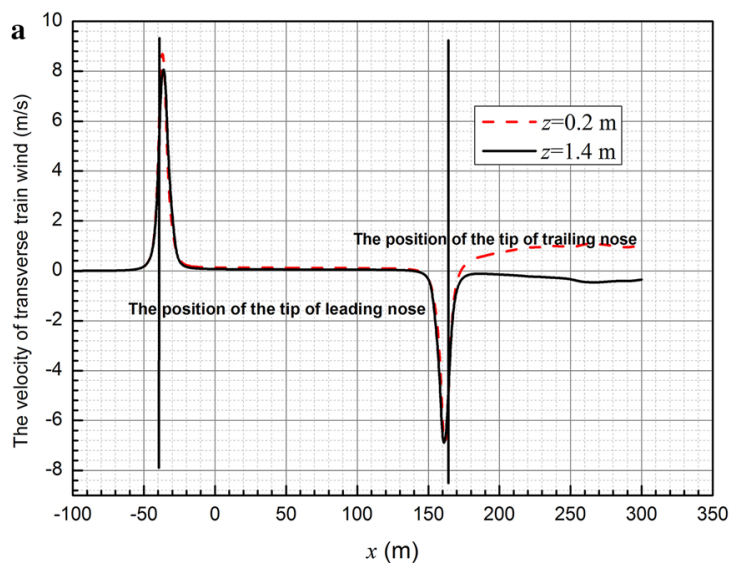

Figures 9 and 10 show the LTW and TTW along the train length illustrated at $3.0 \mathrm{~m}$ from the COT at different distances from the ground for 8-car group and 16-car group trains. It can be seen that regardless of the LTW or the TTW in the upstream, the same spatial distribution is retained as that of 3-car group train. The magnitude of the train wind remains the same too. The magnitude of the TTW in the downstream remains the same as that of the 3-car group train. The TTW mainly relates to the streamlined shape, seldom to the length of the train. However, the LTW apparently varies for trains with different groups. The maximum LTW reaches $3.5 \mathrm{~m} / \mathrm{s}$ for a 3 -car group, while it is 16.4 and $16.2 \mathrm{~m} / \mathrm{s}$ for 8 -car and 16-car group trains, respectively. This is a result of the development of a boundary layer along the train length. Different lengths of trains lead to different thicknesses of the boundary layer in the downstream, which also leads to different states of trailing vortices. As shown in Fig. 9, a line located $3 \mathrm{~m}$ from the train axis is very close to the edge of the boundary layer of the flow, and it comes into the boundary layer at $x=140 \mathrm{~m}$. As a result, a stronger longitudinal train wind can be observed in these places.

Figure 11 shows the variation in the train wind at lines along the train length ranging from 1.7 to $4.2 \mathrm{~m}$ from the COT at a height of $1.4 \mathrm{~m}$ from the ground. It can be seen that the LTW is almost the same before the streamlined head for different lines varying considerably in the downstream direction. For a line of $y=1.7 \mathrm{~m}$, it is just $0.01 \mathrm{~m}$ from the train side surface and completely inside the boundary layer, which results in a stronger LTW. Because of the sharp variation in the shape at the connection between the streamlined head and the train body, the maximum transverse train wind is observed here. With an increasing cross distance from the train side surface, the LTW begins to decrease. Meanwhile, the LTW tends to be stronger in the downstream direction owing to the boundary-layer effect. Velocity profiles at dif-

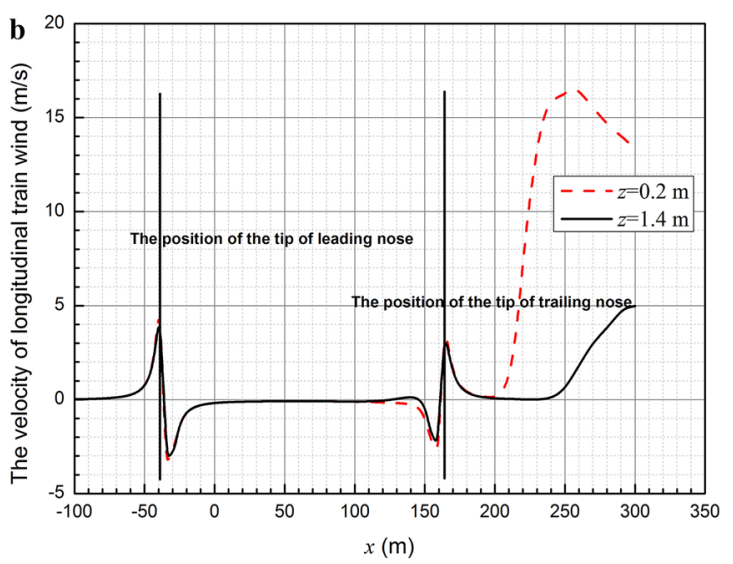

Fig. 9 a TTW of 8-car group simplified train along train length. b LTW of 8-car group simplified train along train length 

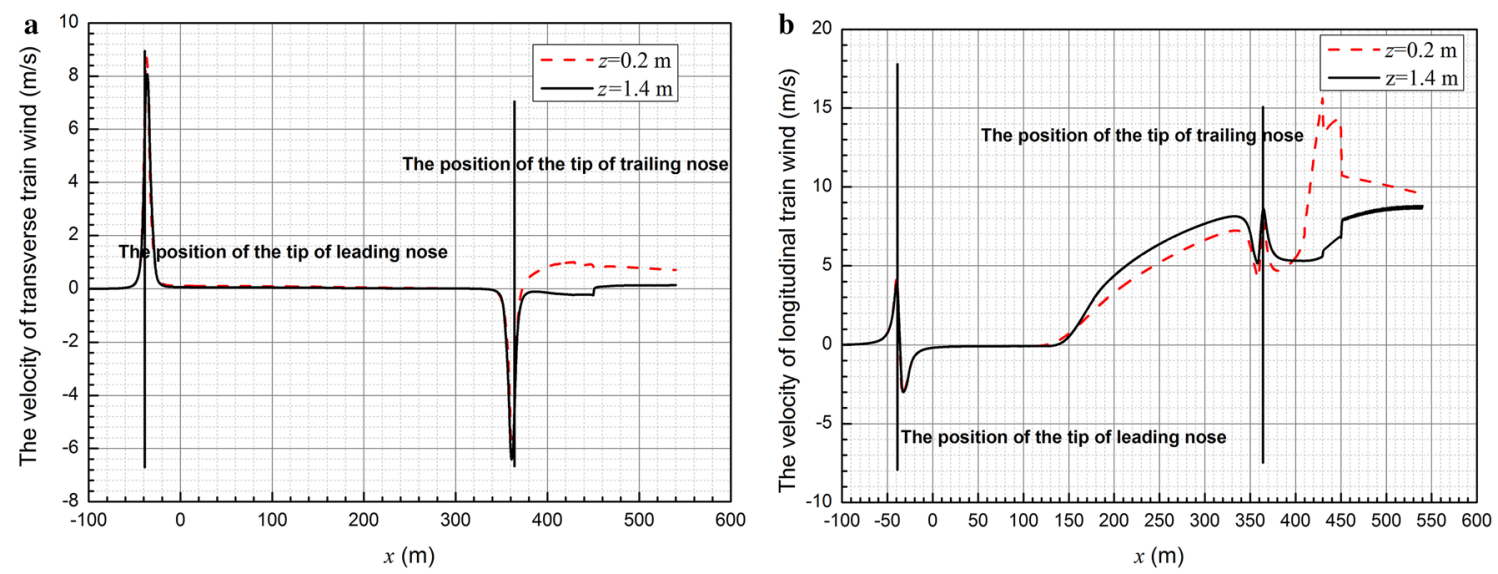

Fig. 10 a TTW of 16-car group simplified train along train length. b LTW of 16-car group simplified train along train length
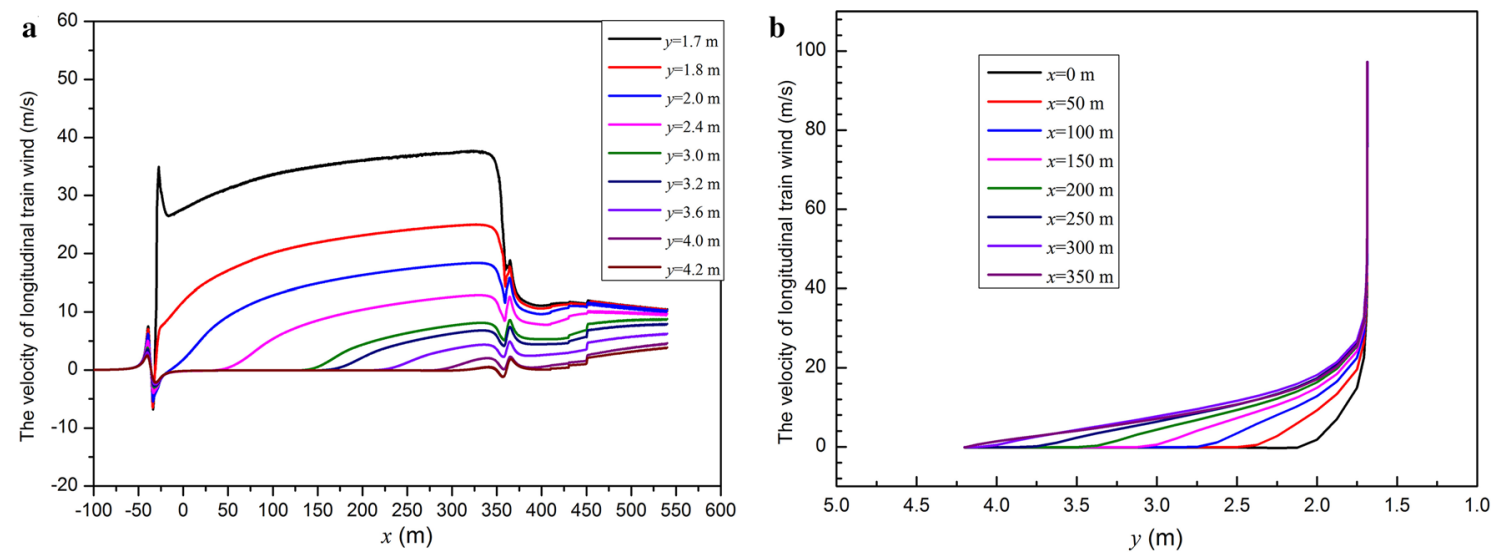

Fig. 11 a LTW of 16-car group simplified train along train length at different distances from COT at $z=1.4$. b LTW distribution at different positions along train length at $z=1.4 \mathrm{~m}$

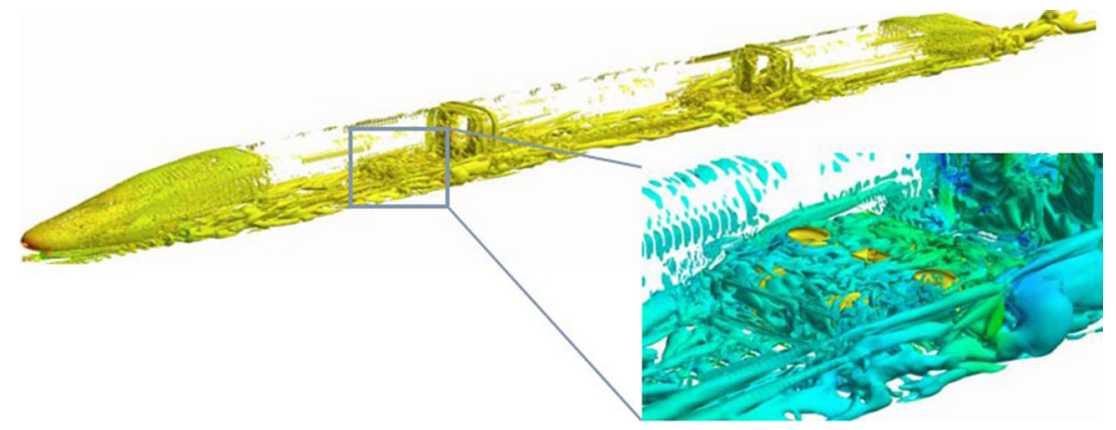

Fig. 12 Isosurface of $Q$ around train flow field

ferent positions along the train body are shown in Fig. 10b, in which the $x$-coordinate denotes the normal distance along the train axis. It can be seen that the longitudinal velocity is increased as the position goes backward, indicating that the boundary layer thickens in the downstream direction.
It can be concluded that a stronger train wind could be induced at the leading and trailing streamline. When a simplified train model is adopted, the model with fewer groups could underestimate the magnitude of the train wind in the downstream direction. 


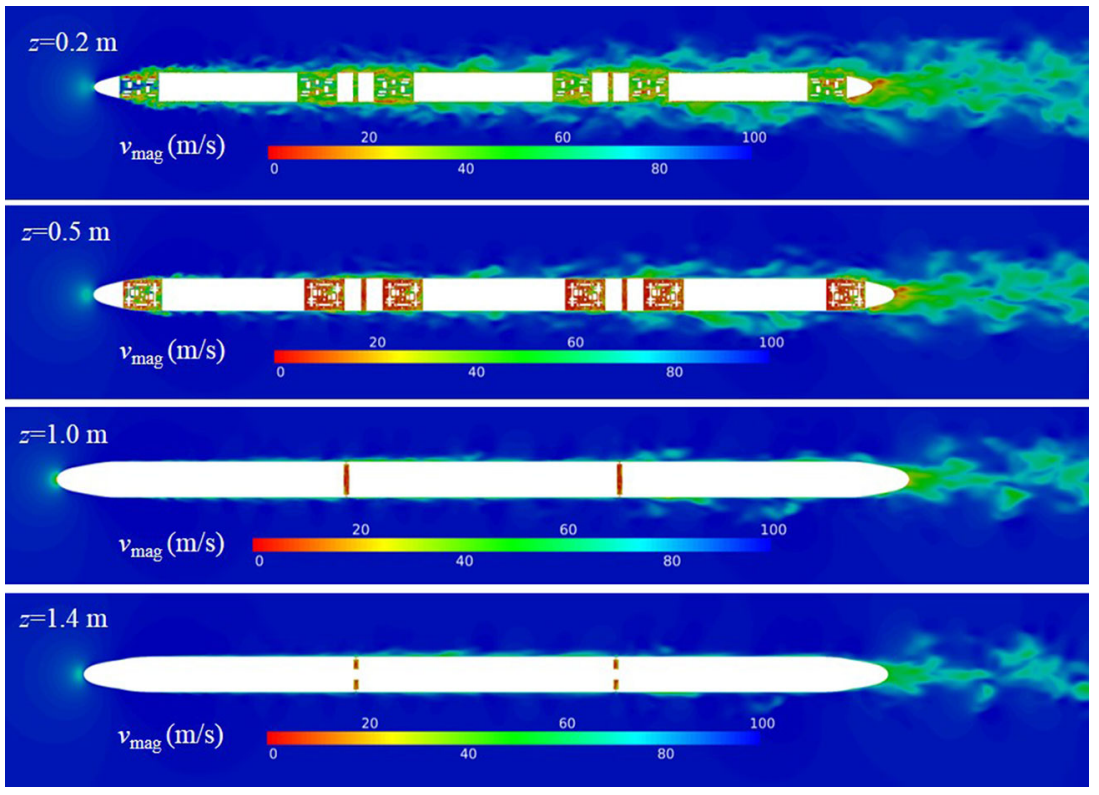

Fig. 13 Instantaneous velocity magnitude contour at different heights
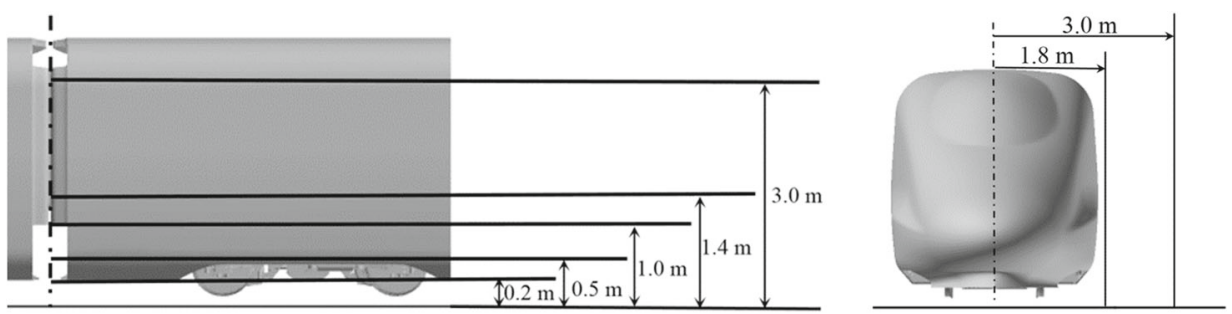

Fig. 14 Schematic diagram of relative train position
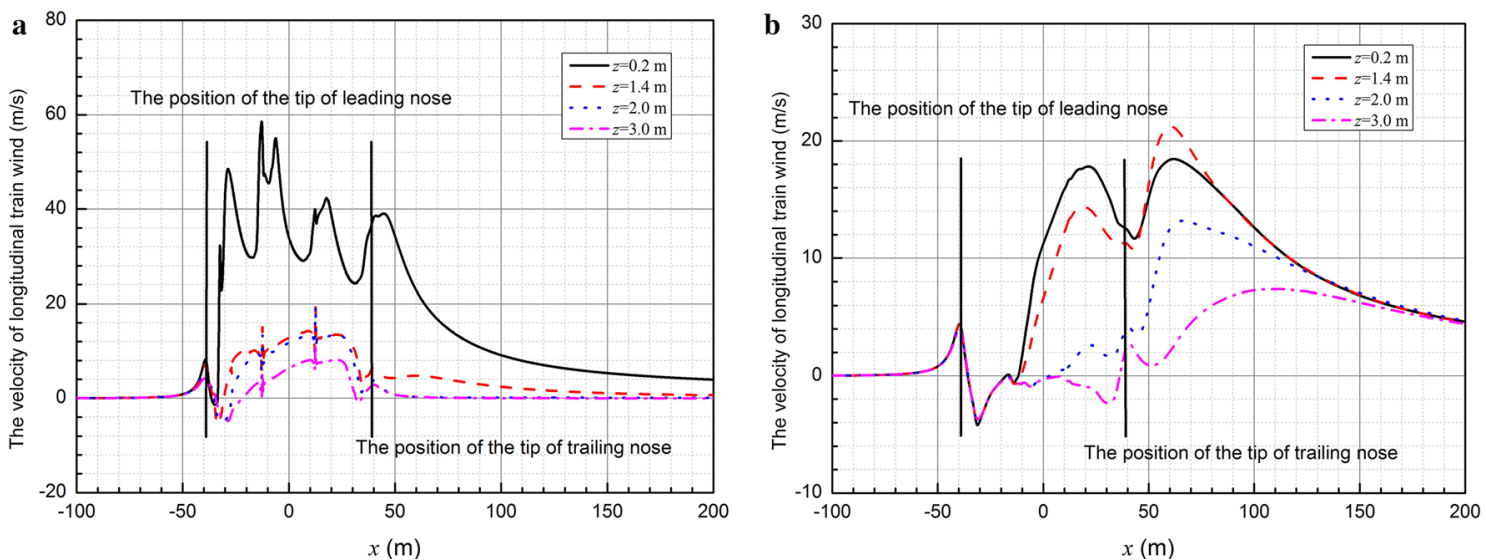

Fig. 15 LTW distribution along real train length for 3-car group. a LTW distribution at $y=1.8 \mathrm{~m}$ from COT. b LTW distribution at $y=3.0 \mathrm{~m}$ from COT

\subsection{Characteristic of train wind of real train model}

When a simplified model is utilized, except for the leading and trailing streamlines, the disturbance to the flow by the train body is very limited. However, when a real train is run- ning, the components will cause a strong disturbance to the flow. These components consist of the bogies and the corresponding bogie cabins in the bottom, the windshields in the middle, and the pantographs on the top of the train. In this section, a real train model is adopted, and all the com- 

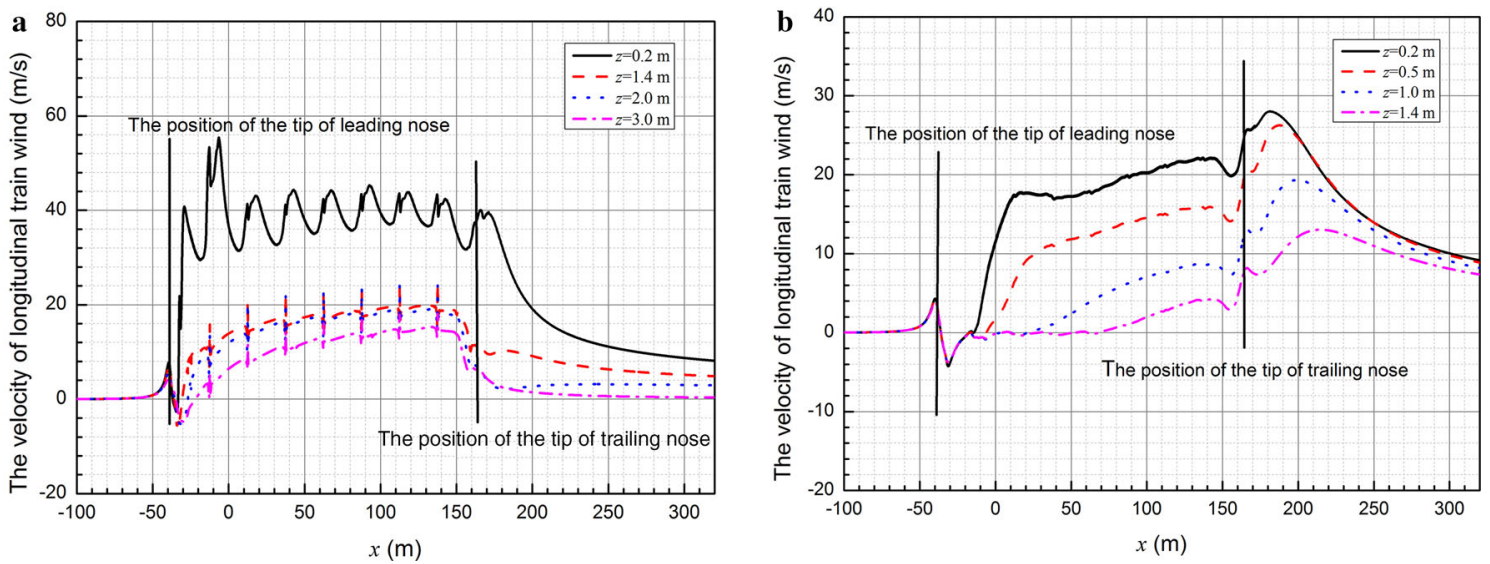

Fig. 16 LTW distribution along real train length for 8-car group. a LTW distribution at $y=1.8 \mathrm{~m}$ from COT. b LTW distribution at $y=3.0 \mathrm{~m}$ from COT
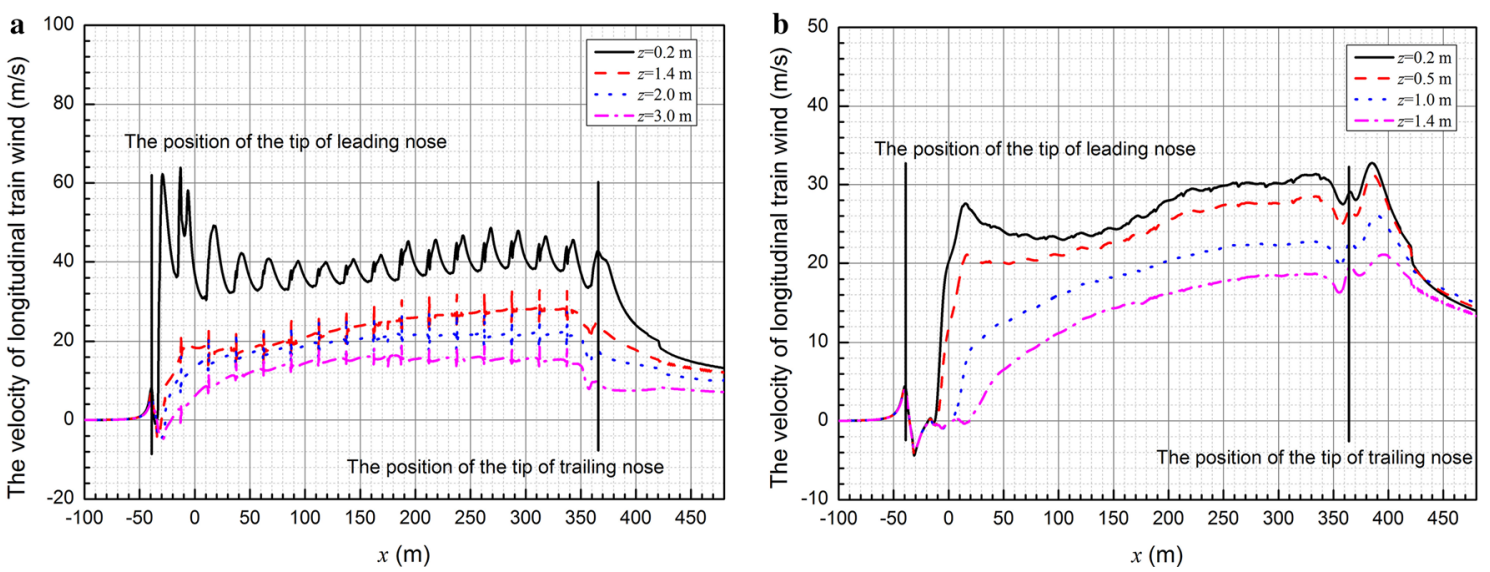

Fig. 17 LTW distribution along train length for 16-car group. a LTW distribution at $y=1.8 \mathrm{~m}$ from COT. b LTW distribution at $y=3.0 \mathrm{~m}$ from COT

ponents are considered except for the pantographs, whose influence on the slipstream around the train body is less pronounced.

Figure 12 shows the isosurface of $Q$ around the train. $Q$ represents the second-order invariant of the velocity gradient tensor and can be used to reveal the vortices in the flow field. It can be seen that strong vortices exist at the bottom of the train, especially in the bogie regions.

Figure 13 shows the velocity contour at different heights around a high-speed train. It can be seen that the flow is disturbed strongly in regions around the bogies and windshields. When the train is running, the bogies and bogie cabins encounter flow around them, and flow inside the bogie cabin is brought about at high speeds. Owing to the sharp curvature of the windshields, flow is disturbed, and consequently the flow inside the boundary layer is changed. The flow is easily separated in the bogie zones, and strong vortices can be observed there. As a result, the surrounding train wind is strengthened. As the height increases, the train wind tends to weaken, which is a result of the relatively lower position of the bogie cover. However, compared to the simplified model, the velocity distribution in the weak zone for the real train model is more complicated since the flow in the weak zone is more turbulent.

Considering the unsteady characteristics of the flow field, the time-averaged train wind at $2 \mathrm{~s}$ is adopted when the train wind is analyzed. Figure 14 shows the positions of the train wind probes compared to the train.

Figures 15, 16, and 17 show the distribution of the LTW along the train body at different heights for the 3-car, 8-car, and 16-car group real train models. The exact positions of the nose tip for the leading and trailing cars are also depicted in the figure. It can be seen that the velocity of the LTW is the same for high-speed trains of different groups in the zone around the leading nose. The train wind decreases as the height increases. Compared to the simplified model, the distribution of wind around the leading nose is the same for the real train model, indicating that the bogie zones have little influence on train wind in the upstream. After the flow 

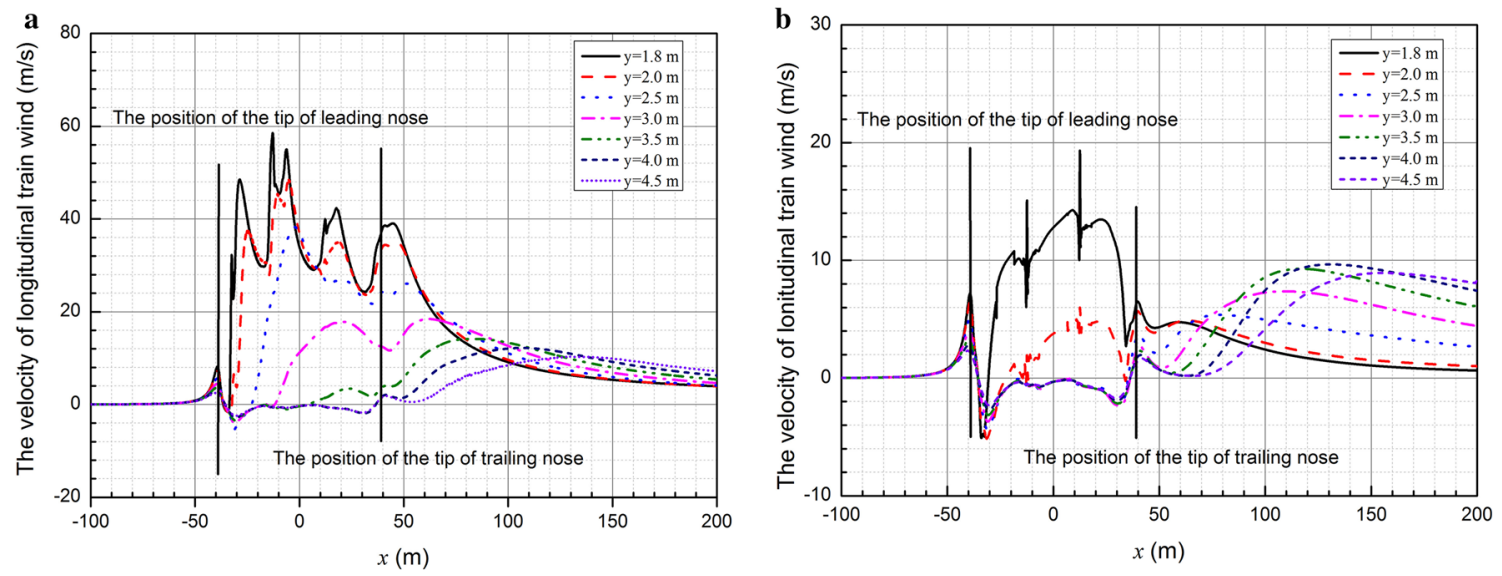

Fig. 18 LTW distribution along real train length for 3-car group. a LTW distribution at different distances from COT at $z=0.2 \mathrm{~m}$. $\mathbf{b}$ LTW distribution at different distances from COT at $z=1.4 \mathrm{~m}$
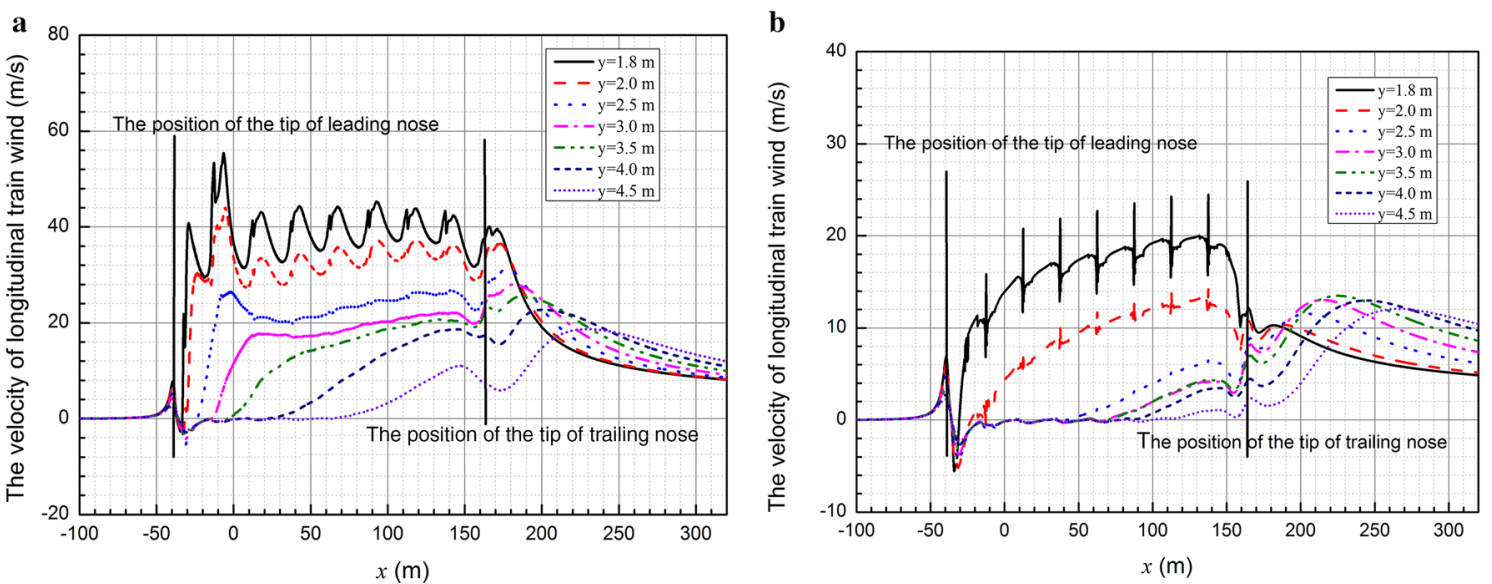

Fig. 19 LTW distribution along real train length for 8-car group. a LTW distribution at different distances from COT at $z=0.2 \mathrm{~m}$. b LTW distribution at different distances from COT at $z=1.4 \mathrm{~m}$
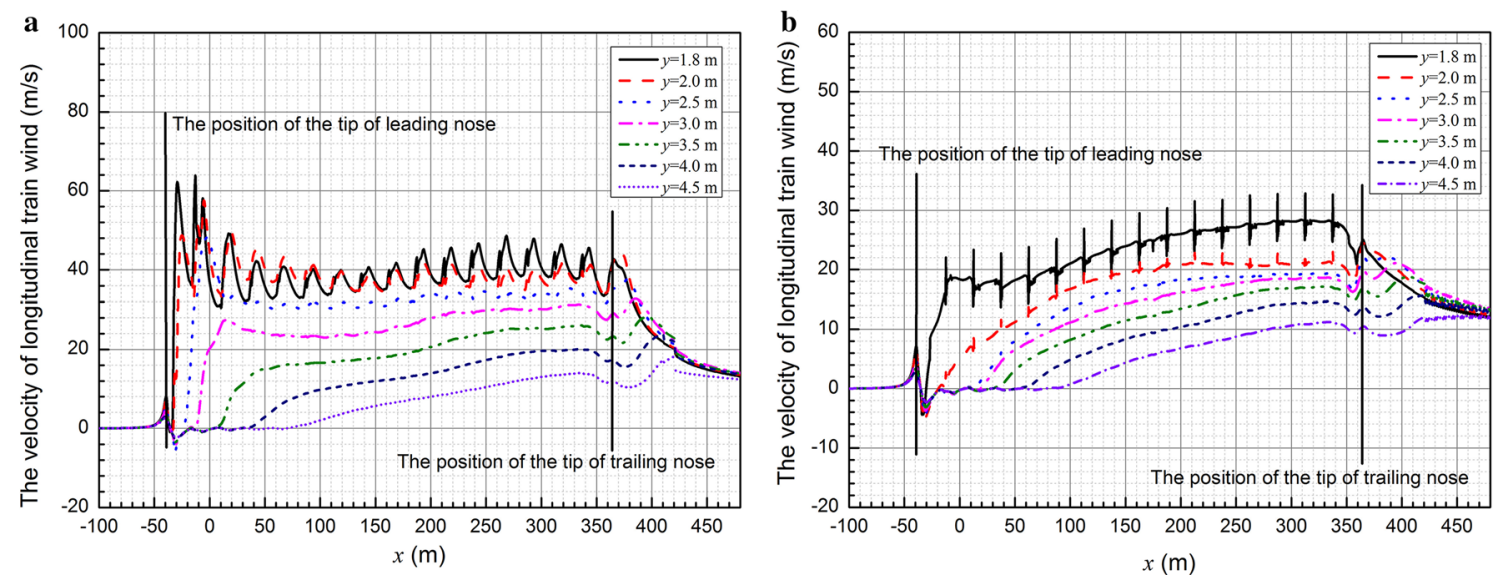

Fig. 20 LTW distribution along real train length for 16-car group. a LTW distribution at different distances from COT at $z=0.2 \mathrm{~m}$. $\mathbf{b}$ LTW distribution at different distances from COT at $z=1.4 \mathrm{~m}$

passes the leading streamline, it is disturbed by the bogies and the bogie cabins. As a result, the velocity distribution inside the boundary layer varies significantly. The difference increases when approaching the ground. Comparing the train models with different groups, the maximum train wind exists around the second bogie zone for a line of $y=1.8 \mathrm{~m}$ and 

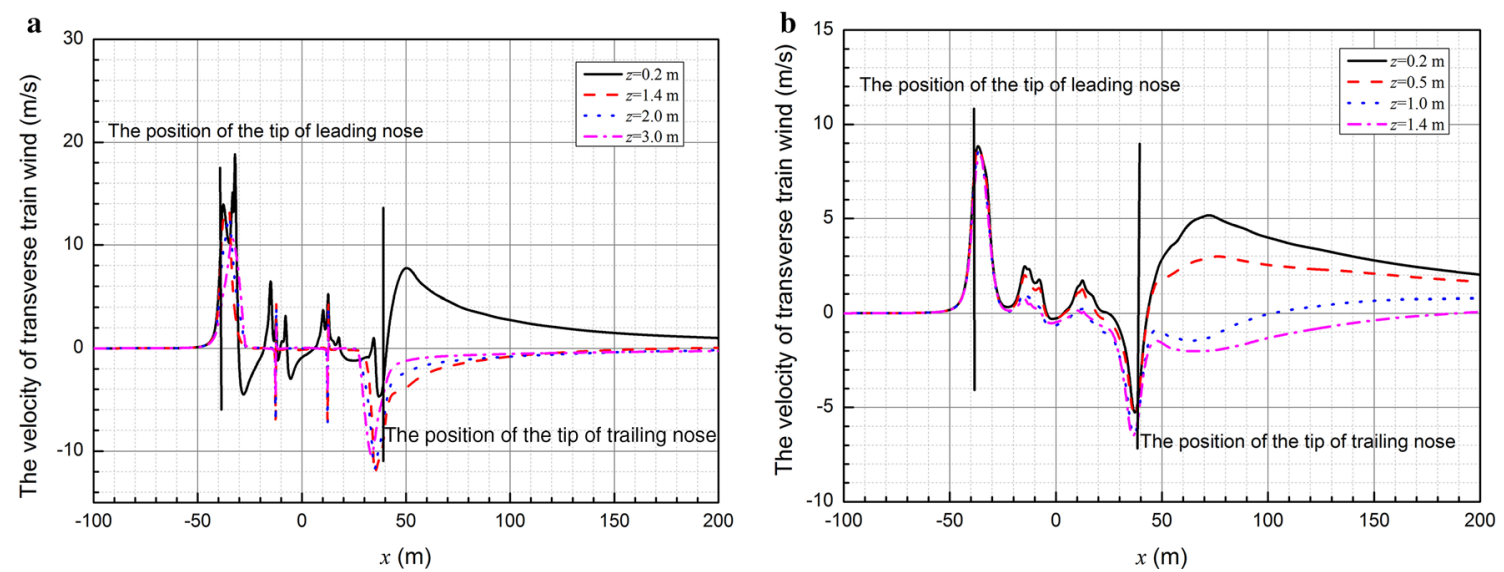

Fig. 21 TTW distribution along real train length for 3-car group. a TTW distribution at $y=1.8 \mathrm{~m}$ from COT. b TTW distribution at $y=3.0 \mathrm{~m}$ from COT
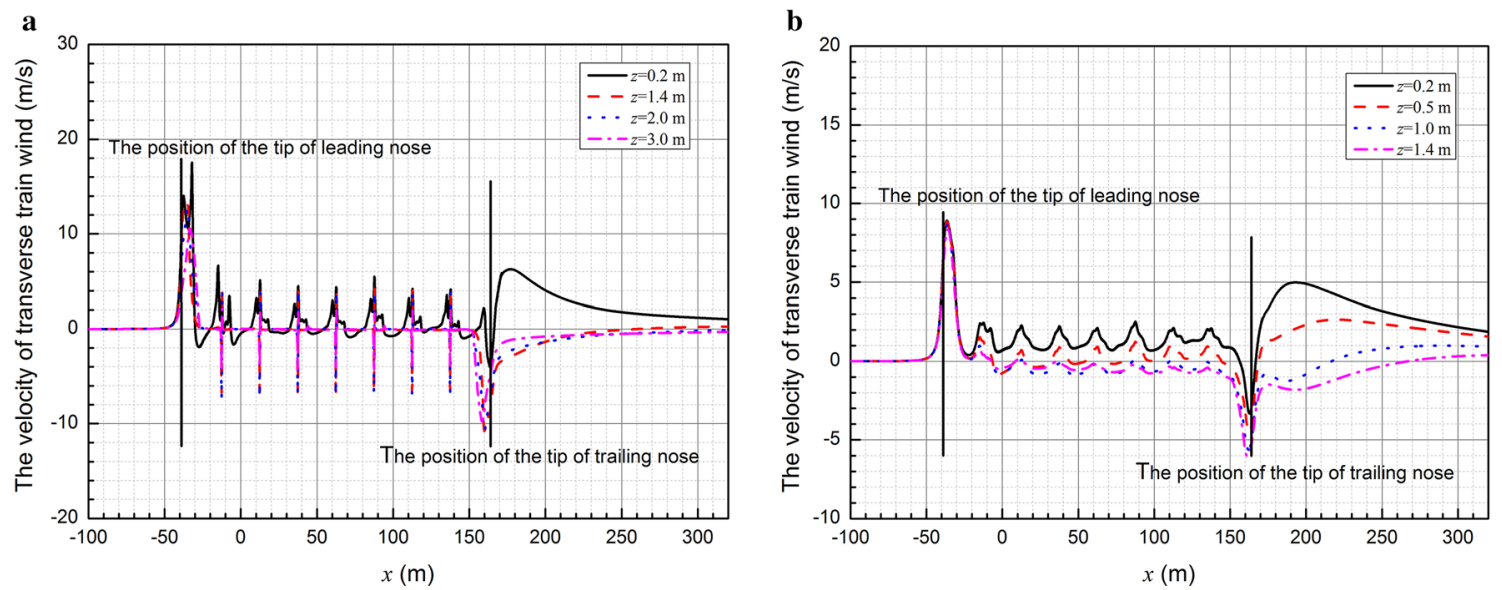

Fig. 22 TTW distribution along real train length for 8-car group. a TTW distribution at $y=1.8 \mathrm{~m}$ from COT. b TTW distribution at $y=3.0 \mathrm{~m}$ from COT
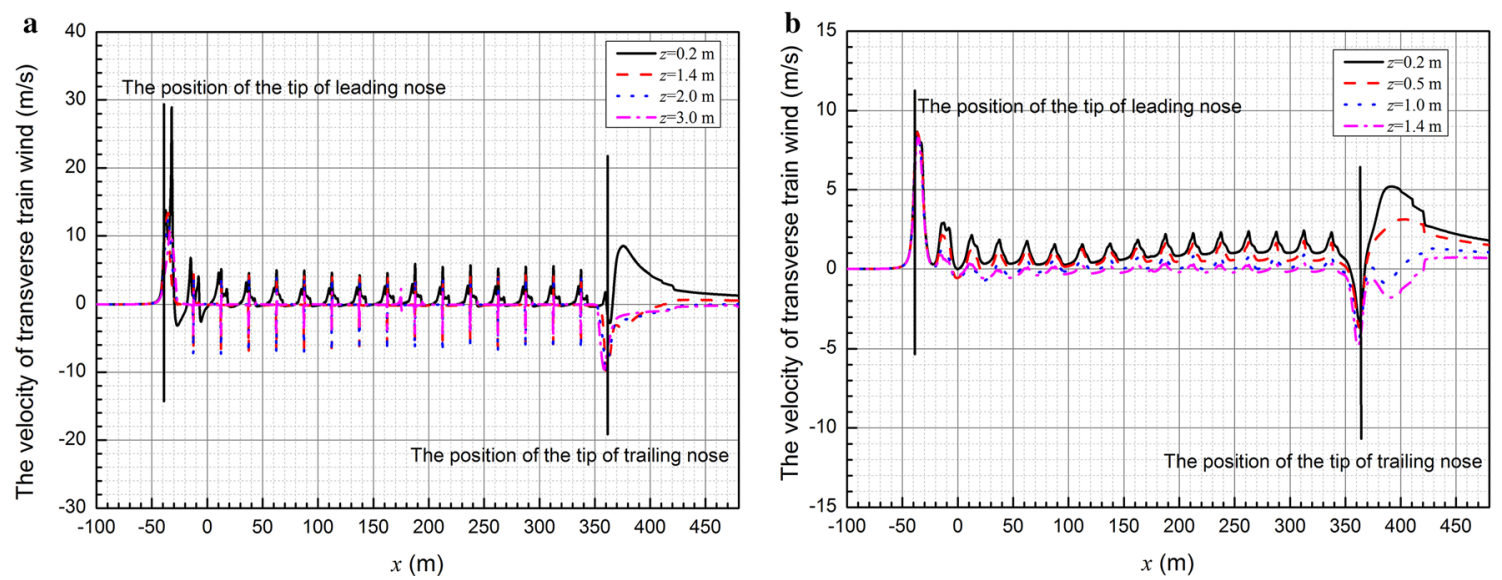

Fig. 23 TTW distribution along real train length for 16-car group. a TTW distribution at $y=1.8 \mathrm{~m}$ from COT. b TTW distribution at $y=3.0 \mathrm{~m}$ from COT

$z=0.2 \mathrm{~m}$. Meanwhile, the value of the maximum LTW is the same compared to each other (3-car, 8-car and 16-car models), since the train wind there is mainly a result of the flow from the first and second bogie zones. For the $y=1.8 \mathrm{~m}$ section plane, as the height increases, the development of the boundary layer begins to dominate instead of the influence 

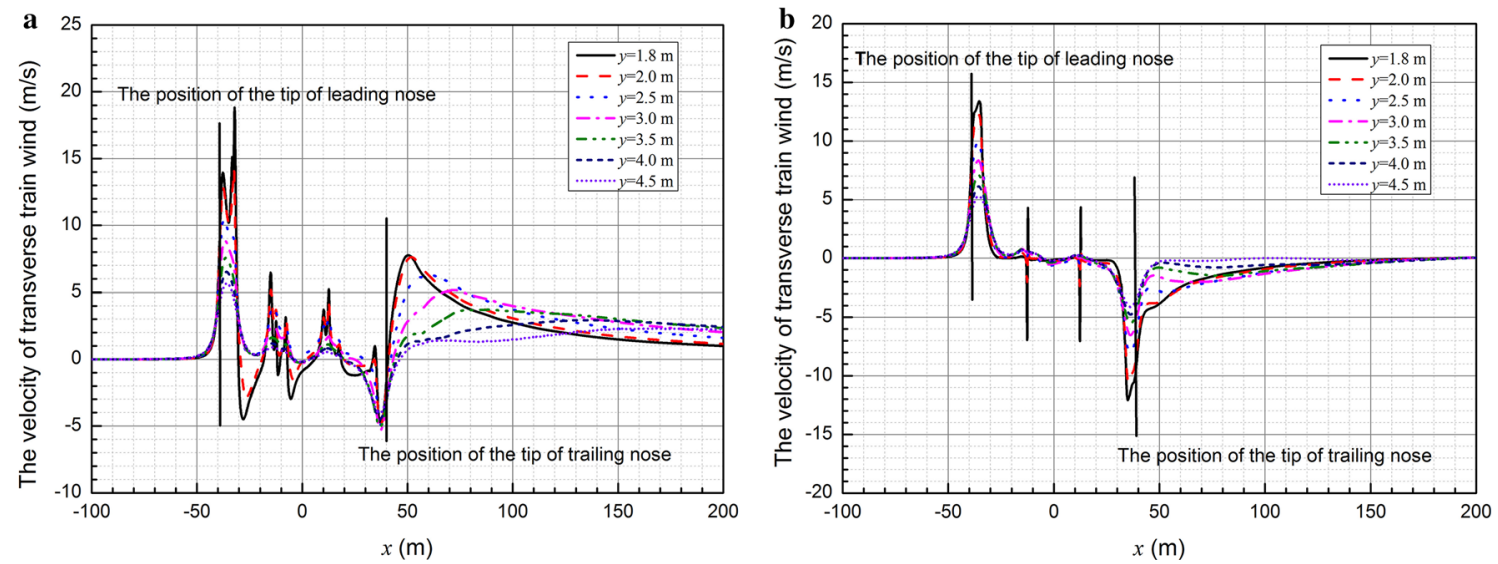

Fig. 24 TTW distribution along real train length for 3-car group. a TTW distribution at different distances from COT at $z=0.2 \mathrm{~m}$. b LTW distribution at different distances from COT at $z=1.4 \mathrm{~m}$
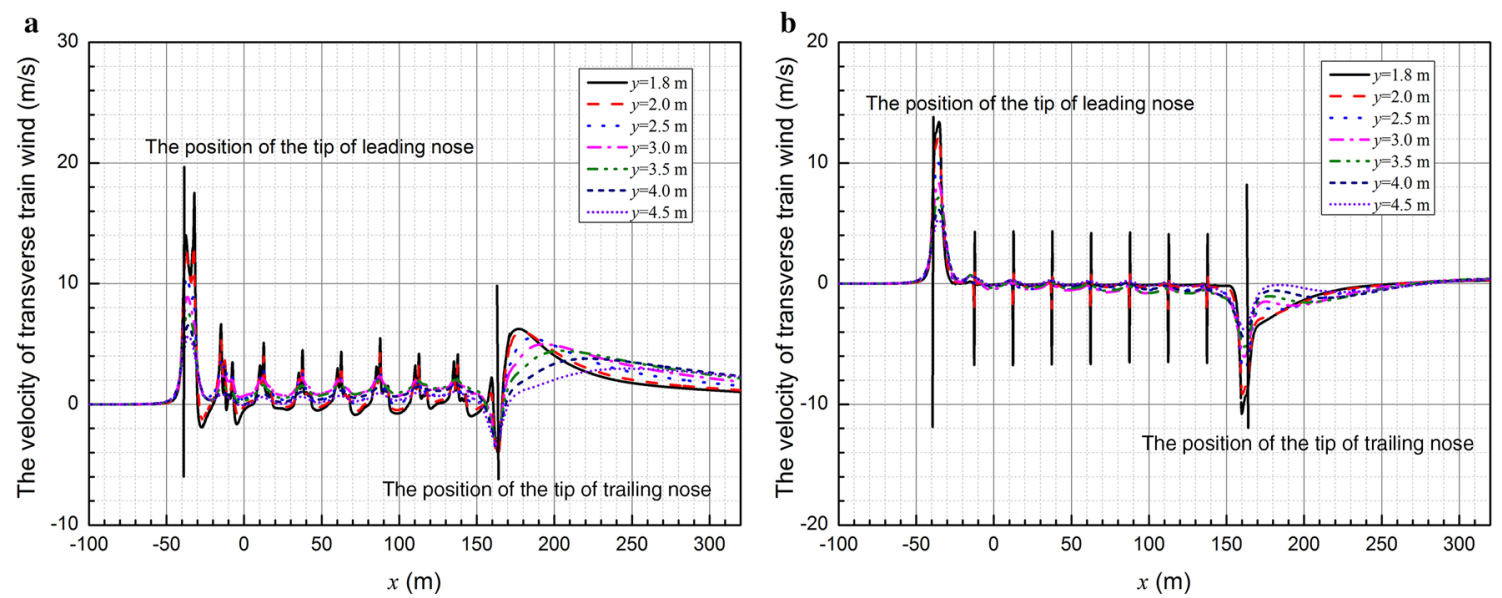

Fig. 25 TTW distribution along real train length for 8-car group. a TTW distribution at different distances from COT at $z=0.2 \mathrm{~m}$. b LTW distribution at different distances from COT at $z=1.4 \mathrm{~m}$

of the bogie region. As the length of the train increases, the maximum value of the velocity of the LTW in the downstream part of the train increases, and the maximum LTW exists at the trailing train body, which is just in front of the trailing streamline.

For places far from the central line of the train $(y=3.0 \mathrm{~m}$, for instance), the influence of the bogie zone becomes limited. Compared to the simplified model, the LTW in the downstream part of the real train model grows much stronger for a line of $y=3.0 \mathrm{~m}$ and $z=0.2 \mathrm{~m}$. At a position of $y=$ $3.0 \mathrm{~m}$ and $z=1.4 \mathrm{~m}$, it will easily enter the boundary layer owing to the influence of the windshields and bogies. Consequently, a strong LTW is observed. This position is nearly outside of the boundary layer for the case of the 3-car group train. For the case of an 8-car group train, this line enters the boundary layer at $x=140 \mathrm{~m}$ for the simplified model and at $75 \mathrm{~m}$ for the real train model. For the case of a 16-car group, the entry spots are the same as with the 8-car group. Analyz- ing the distribution of the LTW in the wake zone, a significant difference can be observed for the simplified model and the real train model, indicating that the structures at the bottom of the train greatly influence the flow status in the wake zone.

Figures 18, 19, and 20 show the distributions of the LTW along the train body at different distances from the central line of the train and different heights. For the $z=0.2 \mathrm{~m}$ section, the tendency is the same for trains with different size groups. As the distance from the central line of the train increases, the LTW decreases. For the position close to the central line, the maximum LTW occurs at the middle of the train. However, as the distance increases, the maximum LTW occurs in the wake zone. For the $z=1.4 \mathrm{~m}$ section, the distribution of the longitudinal train wind along the middle part of the train is the same as in the lower section. However, the maximum LTW of the train with 16 groups is significantly higher than that of the train with 3 groups. 

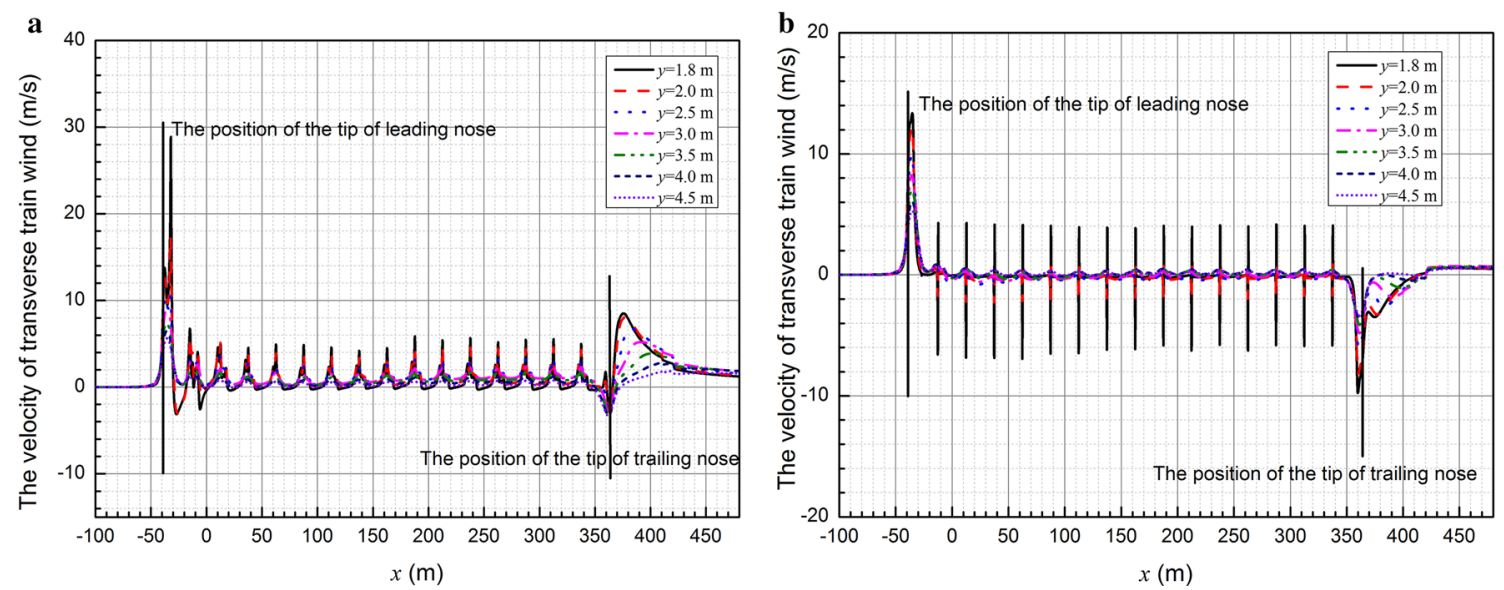

Fig. 26 TTW distribution along real train length for 16-car group. a LTW distribution at different distances from COT at $z=0.2 \mathrm{~m}$. b LTW distribution at different distances from COT at $z=1.4 \mathrm{~m}$

Table 1 Maximum magnitude of train wind for different models

\begin{tabular}{|c|c|c|c|c|c|}
\hline Model & Group & $\begin{array}{l}\text { Distance from } \\
\text { ground }(\mathrm{m})\end{array}$ & $\begin{array}{l}\text { Head region } \\
(\mathrm{m} / \mathrm{s})\end{array}$ & $\begin{array}{l}\text { Middle region } \\
(\mathrm{m} / \mathrm{s})\end{array}$ & $\begin{array}{l}\text { Wake region } \\
(\mathrm{m} / \mathrm{s})\end{array}$ \\
\hline \multirow{6}{*}{$\begin{array}{l}\text { Simplified } \\
\text { train model }\end{array}$} & \multirow[t]{2}{*}{3} & 0.2 & 8.8 & 0.3 & 7.7 \\
\hline & & 1.4 & 8.4 & 0.3 & 7.5 \\
\hline & \multirow[t]{2}{*}{8} & 0.2 & 8.8 & 0.2 & 6.9 \\
\hline & & 1.4 & 8.4 & 0.1 & 7.0 \\
\hline & \multirow[t]{2}{*}{16} & 0.2 & 8.8 & 7.4 & 9.3 \\
\hline & & 1.4 & 8.4 & 8.2 & 10.0 \\
\hline \multirow{6}{*}{$\begin{array}{l}\text { Real train } \\
\text { model }\end{array}$} & \multirow[t]{2}{*}{3} & 0.2 & 8.9 & 17.8 & 19.1 \\
\hline & & 1.4 & 8.5 & 6.5 & 7.5 \\
\hline & \multirow[t]{2}{*}{8} & 0.2 & 8.9 & 22.2 & 28.2 \\
\hline & & 1.4 & 8.6 & 4.3 & 13.1 \\
\hline & \multirow[t]{2}{*}{16} & 0.2 & 8.9 & 29.9 & 26.70 \\
\hline & & 1.4 & 8.5 & 18.2 & 17.9 \\
\hline
\end{tabular}

It can also be seen from the figures that a strong influence from the windshields is observed in the $y=1.8 \mathrm{~m} \mathrm{sec}$ tion.

As discussed earlier, an obvious difference exists for the LTW along the train length between the simplified train and real train models. Moreover, as the group number grows, the maximum LTW increases for the real train model.

Figures 21, 22, and 23 show the TTW for trains with different size groups. It can be seen that the place where the maximum TTW at the same height occurs is the same for trains with different size groups, as is the magnitude of the TTW. The maximum TTW decreases as the height increase in the $y=1.8 \mathrm{~m}$ section. For a line of $y=1.8 \mathrm{~m}$ and $z=0.2 \mathrm{~m}$, the maximum TTW of the real train model emerges at $x=-32 \mathrm{~m}$, which is just behind the first bogie, with a magnitude of $18 \mathrm{~m} / \mathrm{s}$. As the height increases, the TTW at different heights coincides along the train body except for the leading and trailing streamlines. This is because of the limited influence of the bottom bogies. However, for the of $y=3 \mathrm{~m}$ section, which is a little far from the bogie cover, the streamlined shape is the main factor that influences the TTW. The streamline of the train is a kind of slender body that leads to the same position for the maximum TTW. The maximum TTW occurs at $x=-37 \mathrm{~m}$ with a value of $8.5 \mathrm{~m} / \mathrm{s}$. Because of the constant area of the cross section of the train body in the middle, the magnitude of the TTW is very close to zero. Although the boundary layer develops along the train body, the influence on the TTW from the variation of the boundary layer is very slight. In addition, the influence of the windshield is also very slight.

Figures 24, 25, and 26 show the distributions of the TTW along the train body at different distances from the central line of the train and different heights. It can be seen that the TTW around the leading streamline decreases as the distance 
from the central line of the train increases. In the middle of the train, taking the section of $z=0.2 \mathrm{~m}$ as an example, the influence of the bogies diminishes as the cross distance increases. For the $z=1.4 \mathrm{~m}$ section, the influence of the windshields diminishes as the cross distance increases. Meanwhile, in the wake zone of the train, it can be observed that the TTW is not affected by the length of the train. For the $z=0.2 \mathrm{~m} \mathrm{sec}-$ tion, the flow is pushed away by the bogie, which leads to a relatively stronger outward TTW. For the $z=1.4 \mathrm{~m}$ section, which is far from the bogies, the TTW is mainly affected by the trailing vortices in the wake zone and goes inward toward the train.

As discussed earlier, the TTW is mainly affected by the streamlined shape, the bogies, and the windshields. However, it is not affected at all by the length of the train.

Table 1 shows the maximum train wind magnitude in different regions at different heights with different groups. It can be seen that the maximum train wind magnitude in the leading streamline region is almost the same for different groups and different models (simplified model or real train model). In the middle region, owing to the influence of the bogies and windshields, the thickness of the boundary layer varies. Consequently, the maximum train wind clearly varies. In the wake zone, the maximum train wind differs as well. Taking the case of the 3-car group model at the line of $y=3 \mathrm{~m}$ and $z=1.4 \mathrm{~m}$ as an example, the maximum train wind magnitude for the simplified model occurs in the leading streamline region and in the wake region for the real train model; this is true in the case of eight groups as well. However, for the case of the 16-car group model, the maximum train wind magnitude for the simplified model occurs in the wake region and in the middle region for the real train model.

\section{Conclusions}

This study evaluates the influences of affiliated components and length on train wind using an IDDES method. The results can be presented as follows:

(1) In the region ahead of the leading nose, the affiliated components and length of the train have no effect on train wind. Train wind is only a function of the streamline shape of the train nose and the running speed of the train.

(2) The length of the train affects the development of the boundary layer: the longer the length of the train, the thicker the boundary layer in the rear part of the train, which strengthens the LTW at $3.0 \mathrm{~m}$ from the center line of the train at different heights from the ground parallel to the train length. The development of the boundary layer has no effect on the TTW, which is only related to the streamline shape.

(3) Affiliated components, such as bogies, bogie cabins, and windshields, affect the flow field in the near field of the train. Bogies and bogie cabins strengthen the train wind near the ground, and the train wind in the windshield region will be disturbed greatly by the windshields.

(4) The distribution of the LTW for a real train is different from that of the simplified model. The velocity of the LTW in the rear part of the real train model is higher than that of the simplified train model. As the train length increases, the maximum value of the LTW increases.

(5) The train wind will be underestimated when a simplified or short-group train model is used. A real train model with an 8-car grouping is adequate for the evaluation of train wind.

\section{References}

1. Baker, C.J., Sterling, M., Figura-Hardy, G., et al.: The effect of train slipstreams on passengers and trackside workers. In: Proceedings of the 7th World Congress on Railway Research, Monteral, 2006

2. Pope, C.W.: Effective Management of Risk from Slipstream Effects at Trackside and Platforms. Rail Safety and Standards Board-T425 Report, 2007

3. Gilbert, T., Baker, C.J., Quinn, A.: Gusts caused by high-speed trains in confined spaces and tunnels. J. Wind Eng. Ind. Aerodyn. 121, 39-48 (2013)

4. Baker, C.: The flow around high speed trains. J. Wind Eng. Ind. Aerodyn. 98, 277-298 (2010)

5. Baker, C.J., Quinn, A., Sima, M., et al.: Full-scale measurement and analysis of train slipstreams and wakes. Part 1: Ensemble averages. Proceedings of the Institution of Mechanical Engineers, Part F: Journal of Rail and Rapid Transit 228, 451-467 (2014)

6. Yao, S., Sun, Z.X., Guo, D.L., et al.: Numerical studies on wake characteristics of high-speed trains. Acta Mech. Sin. 29, 811-822 (2013)

7. Muld, T.W.: Slipstream and flow structures in the near wake of high-speed trains [Ph. D. Thesis] Stockholm: KTH Aeronautical and Vehicle Engineering, 2012

8. Liao, S., Mosier, P., Kennedy, W., et al.: The aerodynamic effects of high-speed trains on people and property at stations in the Northeast Corridor. US Department of Transportation Federal Railroad Administration, 1999

9. Lei, B., Liu, Y.-G.: Computational analysis on human body aerodynamic force due to train induced air flow. J. China Railw. Soc. 21, 20-23 (1999)

10. Huang, Y., Wu, W., Zhang, H., et al.: Discrete vortex method and its engineering application. J. Basic Sci. Eng. 8, 4 (2000)

11. Tong, B., Xia, N., Li, Q.: Dicrete-vortex method in external flow problems. Adv. Mech. 15, 318-328 (1985)

12. Li, R., Zhao, J., Zhang, S., et al.: Influence of the aerodynamic force to human body near high-speed trains. China Railw. Sci. 28, 22 (2007)

13. Hemida, H., Baker, C.: The calculation of train slipstreams using large-eddy simulation techniques. In: 9th World Congress on Railway Research, Lille, 22-26 May, 2011 
14. Hemida, H.N.: Large-eddy simulation of the flow around simplified high-speed trains under side wind conditions. [Ph. D. Thesis] Chalmers University of Technology, Goteborg (2006)

15. Lee, H.: Assessment of potential aerodynamic effects on personnel and equipment in proximity to high-speed train operations. US Department of Transportation Federal Railroad Administration, 1999
16. Peng, L.-M., Liu, C., Shi, C.-H., et al.: Characteristics of the train wind and analysis of personnel safety in the high-speed railway station. J. Zhengzhou Univ. (Eng. Sci.) 34, 99-102 (2013)

17. Star CCM+ User Guide version 9.04, CD-adapco Corporation 